\title{
PLIF Measurement of Turbulent Diffusion in Drag-Reducing Flow with Dosed Polymer Solution from a Wall*
}

\author{
Masaaki MOTOZAWA**, Taiki KUROSAWA**, Tomohiro OTSUKI**, \\ Kaoru IWAMOTO***, Hirotomo ANDO****, Tetsuya SENDA**** \\ and Yasuo KAWAGUCHI** \\ ** Department of Mechanical Engineering, Tokyo University of Science \\ 2641 Yamazaki, Noda, Chiba, Japan \\ E-mail: motozawa@rs.noda.tus.ac.jp \\ *** Department of Mechanical Systems Engineering, Tokyo University of Agriculture and Technology \\ 2-24-16 Nakamachi, Koganei, Tokyo, Japan \\ **** National Maritime Research Institute \\ 6-38-1 Shinkawa, Mitaka, Tokyo, Japan
}

\begin{abstract}
Experimental investigation of the relationship between mass transfer and turbulent drag reduction of the drag-reducing channel flow with dosed polymer solution from a wall was carried out. Planar laser induced fluorescence (PLIF) measurement was employed to investigate the mass transfer of dosed polymer solution. In addition, the polymer concentration distribution was measured directly by extracting samples from the channel flow (Sampling method). In the PLIF measurement, Reynolds number based on the channel height was set to 20000 and poly(ethylene oxide) was used as a polymer. The polymer solution with a concentration from $10 \mathrm{ppm}$ to 200 ppm was dosed at $3 \mathrm{~L} / \mathrm{min}$ from the whole surface of the wall. As a result, in the case of water flow, dosed dyed water was ejected from the wall and was well diffused by the strong turbulent eddy motion. In contrast, when the polymer solution was dosed from the wall, the diffusion was largely suppressed in the near-wall region and drag reduction occurred. This result indicates that turbulent diffusion was suppressed in the near-wall region and momentum transport in the wall-normal direction was also largely suppressed. Moreover, because the polymer solution could be provided continuously into the channel flow downstream of the leading edge of the blowing wall, the drag reduction rate was reduced downstream. Finally, we estimated the Sherwood number based on the mass transfer logic, and the relationship between the drag reduction and mass transfer was discussed.
\end{abstract}

Key words: Toms Effect, Drag Reduction, Wall Dosing Method, Polymer Solution, PLIF, Mass Transfer, Sherwood Number

\section{Introduction}

The addition of dilute polymer solution to turbulent flow can cause clear reduction in the skin frictional drag. This phenomenon is well known as the Toms effect ${ }^{(1)}$. If this drag-reducing effect could be optimally applied to practical systems, it would lead to significant savings in fuel. For instance, one of the successful applications of drag reduction by polymer solution was in the Trans-Alaska Pipeline ${ }^{(2)}$. By adding a certain amount of polymer solution to the crude oil in the pipeline, the desired discharge of two million barrels

Received 3 Feb., 2012 (No. 12-0090)

DOI: 10.1299/jtst.7.272]

Copyright () 2012 by JSME per day could be achieved without constructing additional pumping stations. The 
drag-reducing effect by polymer solution can be classified into homogeneous drag reduction (e.g. drag reduction in pipes or channels with homogeneous polymer solution) and heterogeneous drag reduction (e.g. drag reduction in boundary layers with polymer solution injected from a slot) ${ }^{(3)}$.

Large numbers of studies on the drag reduction by a homogeneous polymer solution (i.e. homogeneous drag reduction) have been performed for more than 50 years. Virk et al. $(4,5)$ investigated the drag-reducing pipe flow by a homogeneous polymer solution in detail. They obtained a maximum drag reduction of about $75 \%$ compared with the turbulent flow of the solvent. They also pointed out the existence of a maximum drag reduction asymptote for the first time. Following these findings, many researchers have investigated the effect of polymer solution on drag reduction using experiments ${ }^{(6,7)}$ and numerical simulations ${ }^{(8,9)}$.

There has also been intensive research on the drag reduction for external flows (i.e. heterogeneous drag reduction) recently. Regarding heterogeneous drag reduction, one of the most popular methods for achieving drag reduction for an external flow is to inject a polymer solution from slots; we call this method "slot injection" in this paper. There have also been a large number of studies on the drag reduction by slot injection to clarify the relationship among the drag-reducing effect, near-wall turbulent structure, and turbulent diffusion. Tiederman et al. ${ }^{(10)}$ investigated the relationship between the drag reduction and wall-layer structure of the drag-reducing flow by the injection of polymer solution from a slot. They reported that the dimensionless spanwise spacing of wall-layer structure streaks increases and the bursting rate decreases. On the basis of these findings, they found that a polymer is not effective to reduce the skin frictional drag when the polymer is confined to the viscous sublayer, but polymer that exists in the buffer layer $\left(10<y^{+}<100\right)$ has a large effect on the drag reduction. Furthermore, Walker and Tiederman ${ }^{(11)}$ measured the profiles of the instantaneous polymer concentration in the same drag-reducing channel flow by laser induced fluorescence (LIF). They examined the mixing process of the injected polymer solution downstream of the slot, and discussed the relationship between the polymer concentration in the near-wall region and drag reduction. Vdovin et al. ${ }^{(12)}$ measured the local polymer concentration in the turbulent boundary layer by taking the polymer samples from the flow at various points downstream of the slot, and developed scaling laws for the diffusion of wall-injected polymer and phenomenological flow region. In a recent study, Winkel et al. ${ }^{(13)}$ measured the drag reduction rate and the polymer concentration in the near-wall region at a high Reynolds number. They investigated the relationship between the local drag reduction and the polymer diffusion by using the scaling law developed by Vdovin et al. ${ }^{(12)}$ In order to investigate the relationship between turbulent Schmidt number and drag reduction, Somandepalli et al. ${ }^{(14)}$ employed combined particle image velocimetry (PIV) and planar laser induced fluorescence (PLIF) measurements in a polymer drag-reduced turbulent boundary layer. Their experiments were also performed in heterogeneous drag-reducing flow with the injection of polymer solution from a slot. They revealed that the spatial distribution of the injected polymer solution and the drag reduction along the plate developed downstream of the slot. In addition, the turbulent Schmidt number approaches 1, as is expected for a purely Newtonian fluid flow, with lower drag reduction. As drag reduction increases, the turbulent Schmidt number also increases.

As mentioned above, the results of many studies on slot injection have indicated that the polymer in the near-wall region has a very important role in reducing the skin frictional drag. However, polymer injected from a slot is diffused downstream of the slot immediately and drag reduction cannot be sustained over a large region. In order to apply the polymer drag reduction to the external flow, such as the flow around ship hulls, constant dosing of polymer to the turbulent boundary layer from the whole surface of the wall is required. Given this background, we suggested a novel method for reducing the skin frictional drag by dosing the polymer solution from the whole surface of the channel wall ${ }^{(15)}$. We called 
this method "wall dosing method". Figure 1 shows a conceptual diagram of this wall dosing method. In this method, because polymer can be provided downstream continuously in the near-wall region, it is possible that the frictional drag can be reduced more effectively compared with slot injection. We think that this wall dosing method could be used to apply antifouling paint to ship hulls and we are developing a new paint for ship hulls that exhibits the Toms effect by continuously releasing a small amount of polymer ${ }^{(16)}$. This paint has a function of leaching out the polymer and eventually reduces the skin frictional drag owing to the effect of dissolved polymer when the ship is cruising. If this is successful, it will produce great savings in fuel, cost of marine transportation, and travel time.

In our previous study, we measured the skin frictional coefficient with dosing polymer solution from the channel wall. As a result, clear drag-reducing effect was obtained by the wall dosing method and this drag-reducing effect had a close relationship with the mass flux of the dosed polymer ${ }^{(15)}$. In addition, to discuss the turbulent structure near the dosing wall, we measured the instantaneous velocity $u$-v in an $x-y$ plane using PIV in the drag-reducing flow with polymer solution dosed from the channel wall ${ }^{(17)}$. However, the relationship between the mass transfer of polymer solutions and drag reduction has not been clarified by our previous experiments. Therefore, this topic is one of the challenging problems of numerical and experiment study on turbulent transport phenomena because it is significant for both basic studies on turbulence and industrial design on how best to use additives in practical applications.

In this study, we measured the polymer concentration distribution of the drag reducing channel flow with dosed polymer solution from the whole surface of the wall by both PLIF measurement and extraction of polymer samples from the channel flow directly (Sampling method). On the basis of the obtained results, the relationship between turbulent diffusion in the near-dosing-wall region and drag reduction was discussed.

\section{Nomenclature}

$\mathrm{Br} \quad$ Brightness of the induced light

$C \quad$ Weight concentration of polymer at each measurement point, (ppm)

$C^{+} \quad$ Non-dimensional concentration

$C_{0} \quad$ Weight concentration of polymer solution for dosing from the wall, (ppm)

$C_{w} \quad$ Weight concentration of polymer at the internal surface of dosing wall, (ppm)

DR Drag reduction rate, $(\%)$

$H \quad$ Channel height, (m)

$\dot{m} \quad$ Mass flux of polymer, $\left(\mathrm{mg} /\left(\mathrm{min} \cdot \mathrm{m}^{2}\right)\right)=C_{0} Q / S$

$Q \quad$ Dosing rate, $(\mathrm{L} / \mathrm{min})$

Re Reynolds number, $=U_{b} H / v$

$S \quad$ Total area of dosing wall, $(0.45 \mathrm{~m} \times 0.45 \mathrm{~m} \times 3)$

Sc Schmidt number

Sh Sherwood number

St Stanton number

$U_{b} \quad$ Bulk mean velocity, $(\mathrm{m} / \mathrm{s})$

$u_{\tau} \quad$ Frictional velocity, $(\mathrm{m} / \mathrm{s})$

$x \quad$ Streamwise distance from the leading edge of the dosing wall, (mm)

$y \quad$ Wall-normal distance, $(\mathrm{mm})$

$y^{+} \quad$ Wall-normal distance normalized by the frictional velocity

$v \quad$ Kinematic viscosity of the solvent, $\left(\mathrm{m}^{2} / \mathrm{s}\right)$

$\rho \quad$ Density, $\left(\mathrm{kg} / \mathrm{m}^{3}\right)$

$\tau_{\text {dosing }} \quad$ Wall shear stress of the water flow with blowing polymer solution, $(\mathrm{Pa})$

$\tau_{\text {water }} \quad$ Wall shear stress of the water flow without blowing polymer solution, $(\mathrm{Pa})$ 


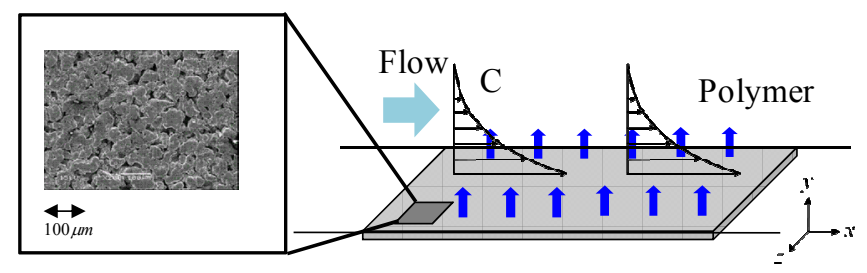

Fig. 1 Conceptual diagram of the wall dosing method.

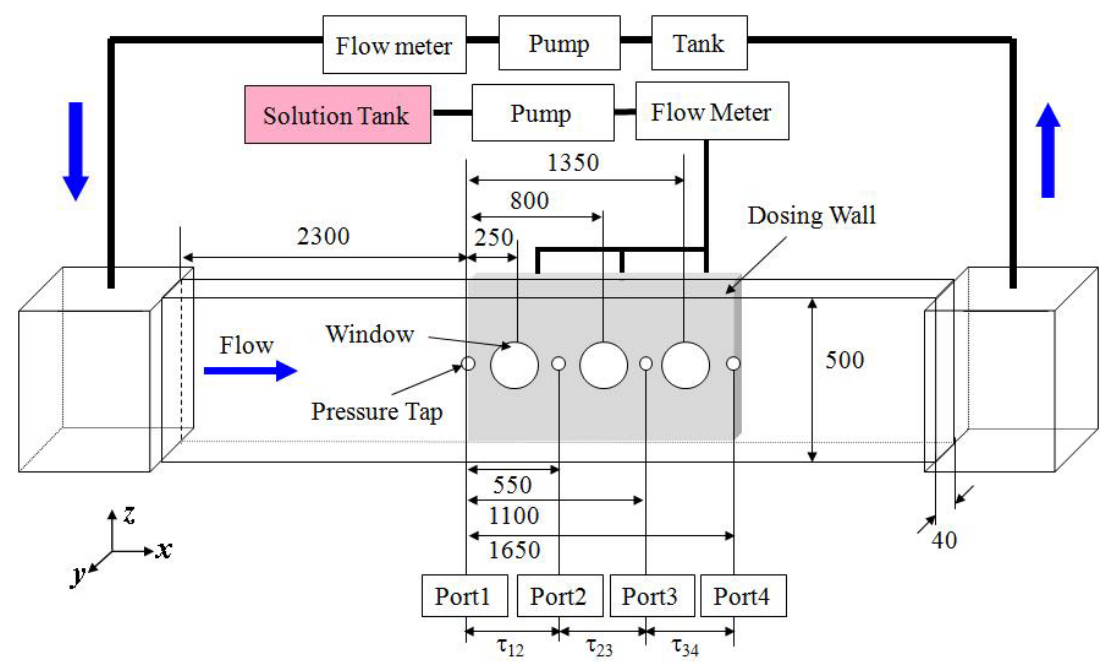

Fig. 2 Experimental facility.

\section{Experiment}

\subsection{Experimental apparatus}

Figure 2 shows a schematic diagram of our experimental facility. The experiments were performed with a closed-circuit water loop having a two-dimensional channel as a test section. This channel is made of transparent acrylic resin, with a length of $6000 \mathrm{~mm}$, a width of $500 \mathrm{~mm}$, and a height of $40 \mathrm{~mm}$. A honeycomb rectifier with a grating space of 10 $\mathrm{mm}$ was set at the channel entrance to remove large eddies. The fluid was circulated by a centrifugal pump and the flow rate could be adjusted by an inverter controlling the rotational speed of the pump motor. An electromagnetic flow meter with an uncertainty of \pm $0.01 \mathrm{~m}^{3} / \mathrm{min}$ was installed downstream of the pump to measure the flow rate. A storage tank was equipped with a heater and an agitator to adjust the temperature of the fluid. The temperature of flowing fluid was kept stable at $25 \pm 0.1{ }^{\circ} \mathrm{C}$ during the experiments.

The wall for dosing of polymer solution was made of sintered porous metal, $450 \mathrm{~mm} \times$ $1350 \mathrm{~mm}$ in size. Pore size of this porous metal wall was $2 \mu \mathrm{m}$. An enlarged image of this porous metal is shown in Fig. 1. This dosing wall was attached to one side of the channel. The leading edge of the dosing wall was located $2300 \mathrm{~mm}$ downstream from the entrance of the channel. Polymer solution can be dosed into the channel flow from the whole surface of this dosing wall. The dosing rate of polymer solution can be adjusted by the pump and the flow meter, which are equipped with a dosing system.

We defined the position of the leading edge of the dosing wall as $x=0$. There were four pressure taps at the opposite side of the dosing wall. These taps were located at $x=0$ (port 1), $x=550 \mathrm{~mm}$ (port 2), $x=1100 \mathrm{~mm}$ (port 3) and $x=1650 \mathrm{~mm}$ (port 4) downstream from the leading edge. Static pressure gradient $(\Delta p)$ between two taps can be measured. The wall shear stress upon dosing of polymer solution can be estimated by the pressure gradient and the force balance as follows: 


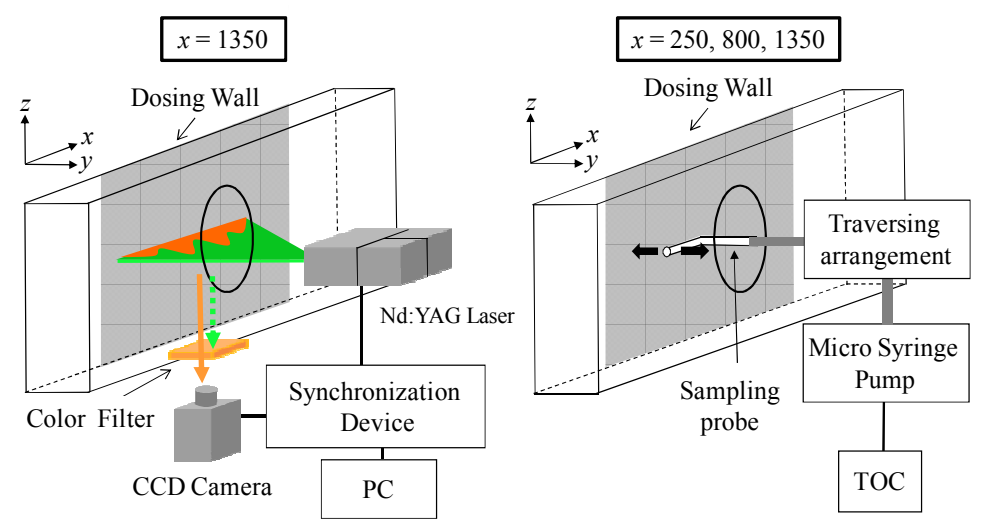

Fig. 3 Experimental apparatus: (a) PLIF system arrangement and (b) Sampling method arrangement.

$$
\tau_{\text {dosing }}=2 \cdot \frac{H}{2 L} \Delta p-\tau_{\text {water }}
$$

where $\tau_{\text {dosing }}$ and $\tau_{\text {water }}$ are the wall shear stress with and without dosing of polymer solution at the same Reynolds number, respectively. In Eq. (1), the wall shear stress of the side wall opposite the dosing wall was estimated to be the same as the skin friction of the water flow $\left(\tau_{\text {water }}\right)$. In this experiment, because there are four pressure taps on the channel wall, we can calculate the drag reduction at various sections. The drag reduction rate is defined by the following equation:

$$
D R=\frac{\tau_{\text {water }}-\tau_{\text {dosing }}}{\tau_{\text {water }}} \times 100(\%)
$$

Reynolds number was defined on the basis of the channel height and the bulk mean velocity $U_{b}$ as follows:

$$
R e=\frac{U_{b} H}{v}
$$

where $v$ is kinematic viscosity of the solvent. In this study, Reynolds number was set to 20000 .

\subsection{PLIF system arrangement}

Figure 3(a) shows the PLIF system arrangement, consisting of a single-pulse laser, laser sheet optics, CCD camera, and synchronizer. The single-pulse laser (produced by New Wave Research Co. Ltd., MiniLase-II/30Hz) was a Nd-YAG laser, having an output of 30 $\mathrm{mJ} /$ pulse and a wavelength of $532 \mathrm{~nm}$. The synchronization device communicated with the CCD camera and computer, which generated pulses to control the single-pulse laser. The CCD camera had a resolution of $2048 \times 2048$ pixels and a pixel pitch of $7.4 \times 7.4 \mu \mathrm{m}$. The camera lens had a focal length of $60 \mathrm{~mm}$ and an aperture of 2.8 .

We used this PLIF system to measure the instantaneous concentration distribution in the $x-y$ plane. The measurement point for PLIF was located at $x=1350 \mathrm{~mm}$. Statistic analysis was performed for 300 concentration fields. Rhodamine-WT (non-toxic and non-carcinogenic dyes) used in this PLIF measurement was a dye that fluoresces under 532 $\mathrm{nm}$ light. We removed the light emitted from the laser with a wavelength of $532 \mathrm{~nm}$ using a color filter, which can block light with a wavelength of less than $550 \mathrm{~nm}$. Therefore, we can measure only the light induced from the dye with a wavelength of $580 \mathrm{~nm}$. We confirmed that the brightness value of the induced light from the dyed water was proportional to the dye concentration in a preliminary experiment. In the PLIF experiments, Rhodamine-WT concentration of the dyed solution for dosing into the channel flow was fixed at $4 \mathrm{ppm}$. 


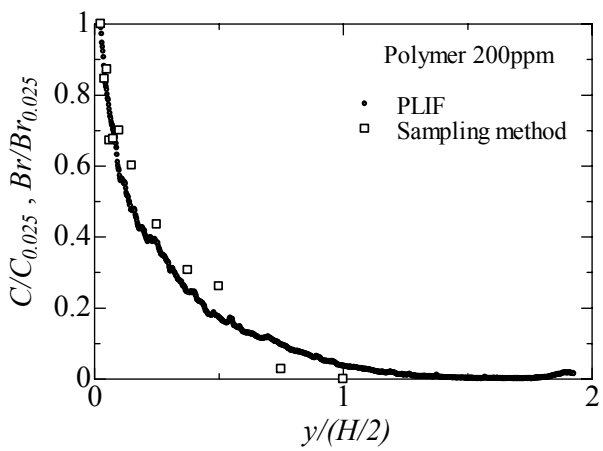

Fig. 4 Mean concentration distribution measured by both PLIF and the Sampling method.

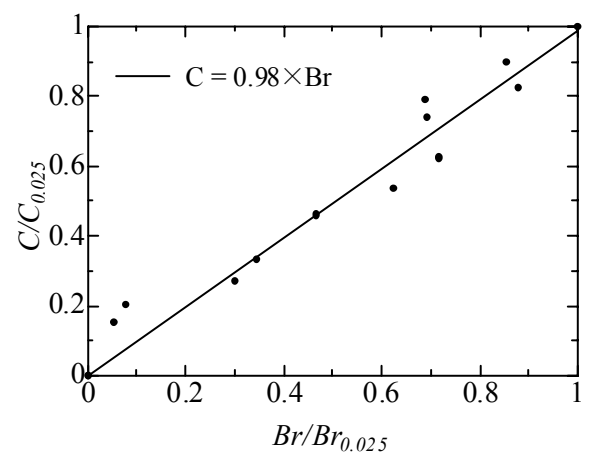

Fig. 5 Relationship between brightness of the induced light and concentration of the dosed polymer solutions.

\subsection{Sampling method}

In contrast to the PLIF measurement, we extracted the flow liquid samples containing dosed polymer solution from the channel flow directly, and measured polymer concentration distribution from the dosing wall. The experimental apparatus for the sampling method is shown in Fig. 3(b). This apparatus consists of a sampling probe, a micro-syringe pump, and a one-dimensional traverse system. A test sample can be extracted directly using the sampling probe and the micro-syringe pump. The sampling probe was fixed on the traverse system. Therefore, test sample can be extracted normal to the dosing wall from $y=0.5 \mathrm{~mm}$ to $y=20 \mathrm{~mm}$ at three streamwise locations of $x=250 \mathrm{~mm}, 800 \mathrm{~mm}$, and $1350 \mathrm{~mm}$. The sampling probe has an internal diameter of $0.7 \mathrm{~mm}$ and an external diameter of $1.0 \mathrm{~mm}$. We extracted a test sample at a speed equal to the velocity distribution in the channel using the syringe pump. Polymer concentration of the test samples was measured using the Total Organic Carbon (TOC) analyzer (produced by the Shimadzu, Co.).

The polymer concentration of the test sample was analyzed on the basis of the following procedure ${ }^{(18)}$. The combustion catalytic oxidation mechanism at $680{ }^{\circ} \mathrm{C}$ can efficiently analyze all organic compounds in the test sample. The total carbon content (TC) is obtained using an infrared detector since all the carbon-containing compounds of the sample are converted into carbon dioxide. Among these contents, part of the carbon dioxide is converted from inorganic carbon-containing compounds, and this part is measured as inorganic carbon element (IC). Finally, the TOC content can be determined by subtracting IC from TC to estimate the concentration of the liquid sample.

\subsection{Polymer solution}

The polymer used in this experiment was PEO-18Z (produced by Sumitomo Seika Chemicals Co., Ltd.). The major component of PEO-18Z is 4.3 million molecular weight water-soluble poly(ethylene oxide). The solvent water was filtered using an ion-exchange resin to remove unnecessary electrolyte. We prepared polymer solution at 10, 25, 50100 , and $200 \mathrm{ppm}$ uniform weight concentration $\left(C_{o}\right)$. The dosing rate $(Q)$ of polymer solution from the whole surface of the dosing wall was fixed at $3.0 \mathrm{~L} / \mathrm{min}$. In this condition, dosing velocity was $8.8 \times 10^{-5} \mathrm{~m} / \mathrm{s}$ from the wall. Therefore, the ratio of the dosing velocity to the bulk mean velocity was $2.0 \times 10^{-4}$ for $R e=20000$. Since the dosing velocity was much smaller than the bulk mean velocity of the channel flow, the dosing flow did not influence the channel flow. 
Table 1: Drag reduction rate with dosed polymer solution calculated by the pressure gradient between port 3 and port 4 .

\begin{tabular}{cc}
\hline$C_{o}(\mathrm{ppm})$ & $D R(\%)$ \\
\hline 200 & 37.6 \\
100 & 28.8 \\
50 & 26.1 \\
25 & 20.4 \\
10 & 15.4 \\
\hline
\end{tabular}

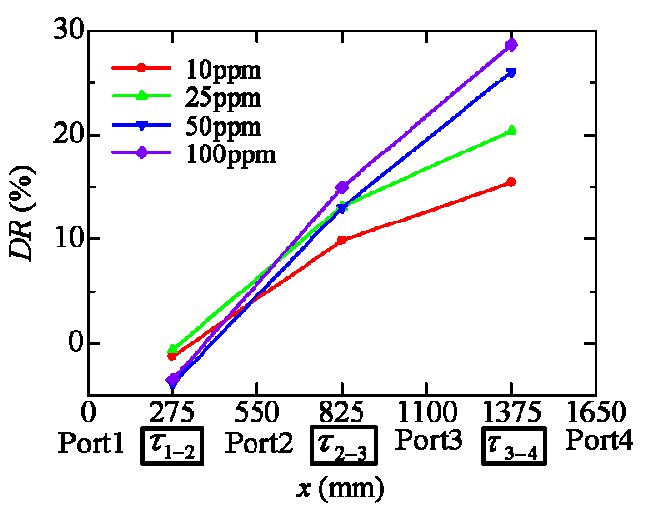

Fig. 6 Streamwise development of the drag reduction rate downstream.

\subsection{Verification of PLIF measurement by the sampling method}

Figure 4 shows the mean polymer concentration distribution measured by the sampling method (plots) and the brightness distribution of the light induced by the laser obtained by the PLIF (solid line). In this varification, the polymer concentration of the dosed polymer solution $\left(C_{0}\right)$ was $200 \mathrm{ppm}$. In the results of PLIF measurements, the vertical axis refers to brightness at each wall-normal position normalized by the brightness measured at $y / h=$ 0.025. In contrast, the vertical axis of the results of the sampling method refers to concentration measured at each wall-normal position normalized by the concentration measured at $y / h=0.025$. The horizontal axis shows the wall-normal position normalized by half of the channel height.

Because the dosed polymer concentration becomes very low (i.e. a few ppm) in the channel owing to the diffusion by channel flow, it is very difficult to measure the polymer concentration distribution directly by the sampling method. In this study, we succeeded in measuring the polymer concentration distribution by the sampling method in only one case for the experimental condition with a dose of $200 \mathrm{ppm}$ polymer solution. However, as shown in Fig. 4, the trend of the mean concentration distribution was almost the same as the trend of the brightness distribution of induced light. This result suggests that the mean concentration distribution can be estimated quantitatively from the PLIF measurements.

Figure 5 shows the relationship between the polymer concentration measured by the Sampling method and the brightness value of the induced light obtained by PLIF at the same wall-normal position constructed from Fig. 4. This figure indicates that the mean concentration of dosed polymer solution has a good agreement with the brightness value obtained by the PLIF measurement. Therefore, we considered it reasonable to undertake quantitative estimation of the polymer concentration distribution by the analysis of PLIF when polymer solutions with different concentrations were dosed from the wall. On the basis of this experiment, the relationship between the brightness value and the weight concentration can be given by the following equation:

$$
C / C_{0.025}=0.98 \times B r / B r_{0.025}
$$

\section{Results and Discussion}

\subsection{Drag reduction rate and streamwise development of drag reduction}

Table 1 lists the drag reduction rate with each concentration of polymer solution dosed from the wall calculated by the pressure gradient between ports 3 and 4 . The drag reduction rate increased with increasing polymer concentration systematically. 


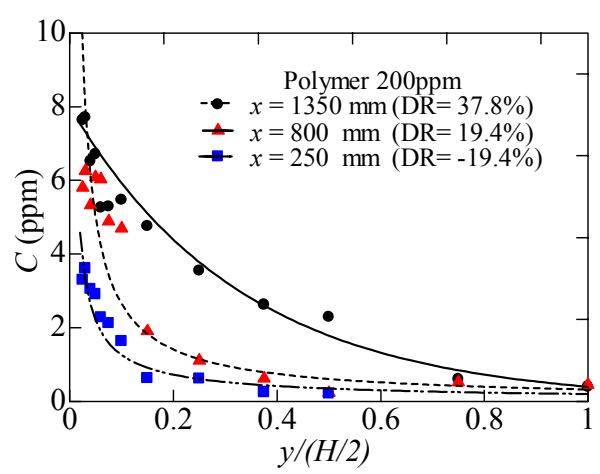

Fig. 7 Streamwise development of polymer concentration boundary layer.

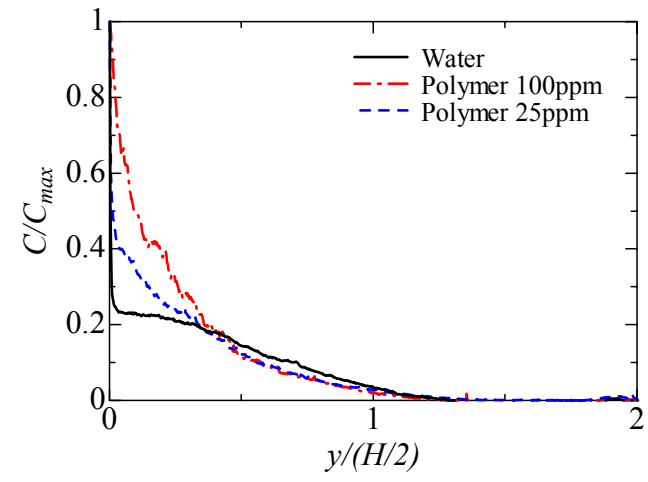

Fig. 8 Mean concentration distribution estimated by PLIF.

Figure 6 shows the streamwise development of the local drag reduction rate downstream of the leading edge of the dosing wall. The drag reduction rate was evaluated locally by the wall shear stress estimated by the pressure gradient between ports 1 and 2 , ports 2 and 3, and ports 3 and 4. This figure indicates that the drag reduction rate develops downstream from the leading edge of the dosing wall. About $37.6 \%$ of the maximum drag reduction was obtained with wall dosing at $x=1375 \mathrm{~mm}$ under the experimental condition in which the length of the dosing wall was $1650 \mathrm{~mm}$. In the wall dosing method, the polymer solution can be provided continuously to the buffer layer and the concentration boundary layer also develops. As a result, the drag-reducing effect also develops downstream.

Compared with the slot injection ${ }^{(19)}$, the drag reduction rate develops within a short distance and is saturated downstream of the slot, but this drag reduction rate cannot be kept at a constant level and gradually decreases. Moreover, to obtain a large drag reduction rate, it is necessary to inject a very high concentration of polymer solution (e.g. 1000 ppm or 500 ppm). According to Poreh and Hsu ${ }^{(20)}$, the trend of the streamwise development of drag reduction can be categorized into the following three regimes in terms of the region downstream from the slot: (i) Development region: the drag reduction develops a maximum drag reduction rate downstream in this region; (ii) Steady region: the drag reduction rate is maintained at a constant level downstream in this region; and (iii) Depletion region: the drag reduction rate decreases downstream in this region. In contrast, in the wall dosing method (this study), the drag reduction rate continues to develop downstream as shown in Fig. 6. In addition, if the dosing wall is longer, the drag reduction rate seems to be saturated and this drag reduction rate can be kept at a constant level downstream of the dosing wall because the polymer solution is provided continuously into the flow downstream. This ability of the wall dosing method is the most significant merit for achieving drag reduction for the external flow. If we can apply the wall dosing method to fairly long walls such as ship hulls, a large drag-reducing effect can be expected using a relatively small amount of polymer.

\subsection{Streamwise development of polymer concentration boundary layer}

Figure 7 shows the distribution of polymer concentration along the wall-normal direction for $R e=20000$ with a dosing concentration of $200 \mathrm{ppm}$ at measurement locations of $x=250 \mathrm{~mm}, 800 \mathrm{~mm}$, and $1350 \mathrm{~mm}$. This distribution was obtained by the Sampling method.

As shown in this figure, a high-polymer concentration layer develops and extends into the outer region downstream owing to the accumulation of polymer solution in the flow direction. This is why the drag reduction rate develops downward along the channel by the 
wall dosing method. The maximum polymer concentration in the near-wall region at $x=$ $800 \mathrm{~mm}$ is similar to that at $1350 \mathrm{~mm}$, and is much higher than that at $x=250 \mathrm{~mm}$. This indicates that, because the polymer solution is provided continuously downstream, the concentration boundary layer develops downstream and polymer remains near the wall. As a result, polymer solution strongly interacts with the coherent structure near the wall as the concentration boundary layer develops downstream. The relationship among the near-wall polymer concentration, mass transfer phenomenon, and drag reduction will be discussed in more detail in later sections.

\subsection{Mean concentration distribution estimated by PLIF}

Figure 8 shows the mean concentration distributions of the dosed polymer solutions with weight concentrations of $25 \mathrm{ppm}$ and $100 \mathrm{ppm}$. These concentration profiles were estimated by the PLIF measurement. In order to confirm the turbulent diffusion of the Newtonian fluid flow, the concentration distribution of the dosed dyed water is also shown in this figure. In the case of dosing polymer solution, there is a region where the concentration is higher than that of the water near the dosing wall $(0 \leqq y / h \leqq 0.4)$. Since the concentration distribution in this region is relatively large, we suppose that the turbulent mass transfer is suppressed by the dosed polymer solution from the channel. Moreover, it can be confirmed that the dosed polymer solution suppresses the diffusivity of the flow liquid close to the wall from the PLIF instantaneous images, as shown in Fig. 14(b). As a result of this suppression, the dosed polymer solution remains in the near-wall region.

Somandepalli et al. ${ }^{(14)}$ conducted combined PIV and PLIF measurements in a flat-plate turbulent boundary layer. Their experiment was performed with slot injection. They revealed that, when the concentration of the injected polymer solution increases, the suppression of the turbulent diffusion becomes strong and the drag reduction rate increases. Our experimental results correspond to their results. However, Walker et al. ${ }^{(11)}$ reported that, when the condensed polymer solution was injected to the turbulent boundary flow from the slot, drag reduction dose not occur, but rather drag increases just downstream of the slot. They concluded that this is because the velocity gradient increases locally owing to the large shear viscosity of the high-concentration polymer solution. In our experiments, we also obtained an increase of drag near the leading edge of the dosing wall, as shown in Fig. 6. In addition, although this finding is not shown in this paper, when the $1000 \mathrm{ppm}$ polymer solution was dosed from the wall, drag reduction did not occur, but rather drag markedly increased. Therefore, we will investigate the drag increase phenomenon upon dosing a high-concentration polymer solution in the future.

\subsection{Analysis of mass transfer}

\subsubsection{Determining Schmidt number from PLIF data}

Figure 9 shows the distribution of the non-dimensional polymer concentration $C^{+}$ versus $y^{+}$compared between the experimental results and the calculated profile obtained by Kader ${ }^{(21)}$. Non-dimensional polymer concentration $C^{+}$is defined by the following equation:

$$
C^{+}=\frac{C_{\mathrm{w}}-C}{\dot{m} / u_{\tau}}
$$

where $\dot{m}$ is the mass flux of dosed polymer solution defined as $\dot{m}=C_{0} Q / S$; here, $S$ is the area of the dosing wall. $C_{w}$ and $C$ are weight concentration of polymer at the surface of the dosing wall and polymer concentration at each position measured by PLIF, respectively. $u_{\tau}$ is the frictional velocity. 


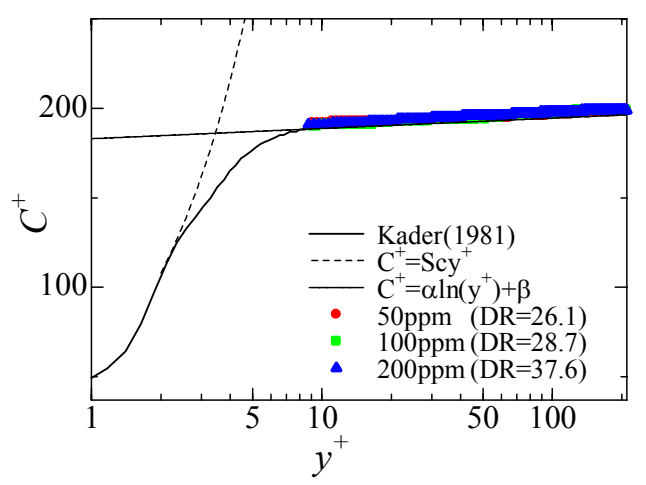

Fig. 9 Non-dimensional concentration distribution compared with the calculation by $\operatorname{Kader}^{(21)}$

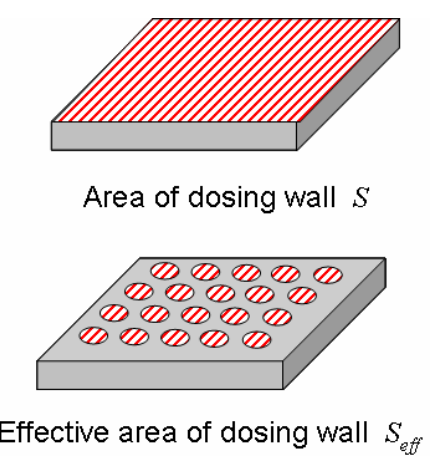

Fig. 10 Conceptual diagram for the explanation of $S$ and $S_{\text {eff }}$

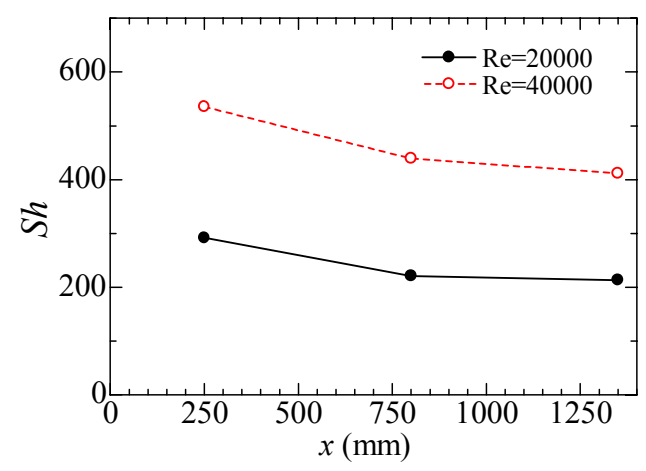

Fig. 11 Relationship between the distance from the leading edge of the dosing wall and Sherwood number.

In this experiment, $C_{w}$ is quite different from $C_{0}$ as shown in Fig. 7. Therefore, we assert the following hypothesis of the relationship between $C_{0}$ and $C_{w}$ based on mass flux conservation:

$$
C_{w}=\frac{A Q}{S u_{\tau}} C_{0}
$$

In this experiment, because polymer solution was dosed from the porous wall, we considered that the real area of the dosing wall $(S)$ is different from the effective area of the dosing wall $\left(S_{\text {eff }}\right)$ as shown in Fig. 9. On the basis of this consideration, we defined the coefficient $A$ as follows,

$$
A=\frac{S_{e f f}}{S}
$$

The meaning of coefficient $A$ is the ratio of the area of the dosing wall to the effective area of the dosing wall. Therefore, coefficient $A$ has the constant value peculiar to the porous wall. $C^{+}$can be represented by Eqs. (6) and (7) as follows:

$$
C^{+}=\frac{C_{0}-C}{\dot{m} / u_{\tau}}+\frac{A Q-u_{\tau} S}{Q}
$$

For different polymer concentrations $C_{o}$ of dosed polymer solution at $R e=20000, C^{+}$ can be estimated by adjusting coefficient $A$ and comparing with the experimental results for 


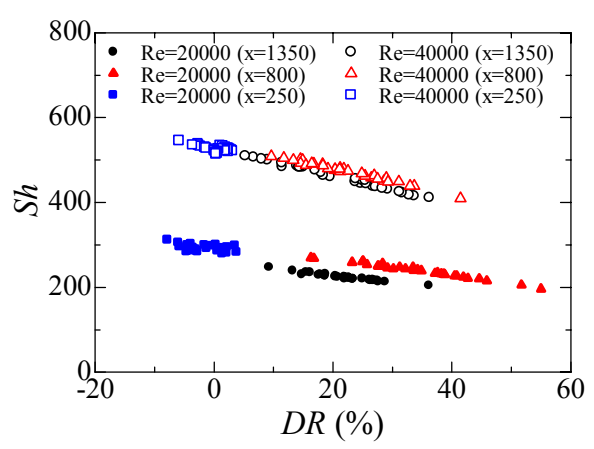

Fig. 12 Relationship between the drag reduction rate and Sherwood number.

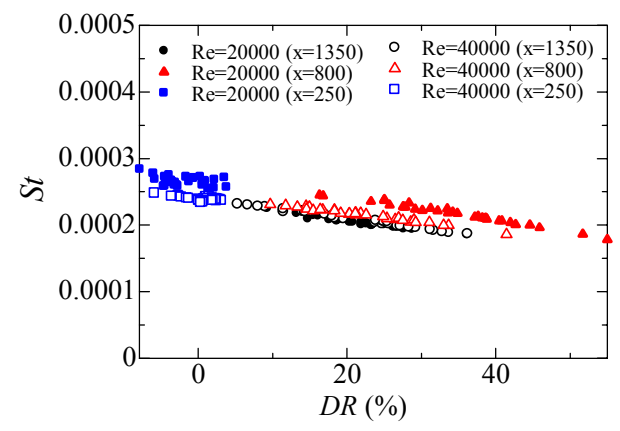

Fig. 13 Relationship between the drag reduction rate and Stanton number.

$C$ with Eq (9). Owing to the similarity of the relationship between heat transfer and mass transfer, the calculation method for temperature distribution is also valid for the concentration distribution with respective changes in notation with a known Schmidt number $(S c)$. According to the calculation method by $\operatorname{Kader}^{(21)}$, the non-dimensional concentration distribution can be expressed as follows:

$$
\begin{array}{ll}
C^{+}=S c y^{+} & \text {(Viscous sub-layer) } \\
C^{+}=\alpha \ln y^{+}+\beta(S c) & \text { (Logarithmic sub-layer) }
\end{array}
$$

Upon adjusting the Schmidt number $S c$ and the coefficient $A$, we make the non-dimensional concentration distribution estimated from PLIF data. This result agrees well with the profile calculated by Kader, as shown in Fig. 9. Value of $S c$ of 55 and $A$ of about 200 are obtained on the basis of the PLIF results.

Finally, because the Schmidt number and $C_{w}$ were obtained, we can calculate the Sherwood number $(S h)$ using experimental data. $S h$ is defined as follows:

$$
S h=\frac{H(-\partial C / \partial y)_{w}}{C_{\infty}-C_{w}}=\frac{\dot{m} H S c}{C_{w} v}
$$

\subsubsection{Extension to other experimental results}

In our previous study, we measured the drag reduction with dosed polymer solution under various flow conditions and dosing conditions. For instance, Reynolds number was set to 20000 and 40000 , the dosing condition was $C_{0}=10,25,50$, and $100 \mathrm{ppm}$, and $Q=$ $0.5,1.0,1.5,2.0,2.5,3.0,3.5$, and $4.0 \mathrm{~L} / \mathrm{min}$. As we assumed that $S c$ is constant under various dosing conditions, we can calculate $C_{w}$ and $S h$ for other cases without a PLIF experiment.

Figure 11 shows the relationship between the distance from the leading edge of the dosing wall $(x)$ and Sherwood number estimated from the results for $R e=20000$ and 40000 with dosed $C_{0}=100 \mathrm{ppm}$ polymer solution at $Q=3 \mathrm{~L} / \mathrm{min}$. This figure indicates that the Sherwood number decreases from the leading edge of the dosing wall downstream. This means that the turbulent diffusion is suppressed downstream because polymer solution accumulates downstream. In addition, the estimated Sherwood number for $R e=40000$ is larger than that for $R e=20000$. Considering the heat transfer analogy in the drag-reducing flow ${ }^{(22)}$, this tendency of the Sherwood number is very suitable for the wall dosing system. 


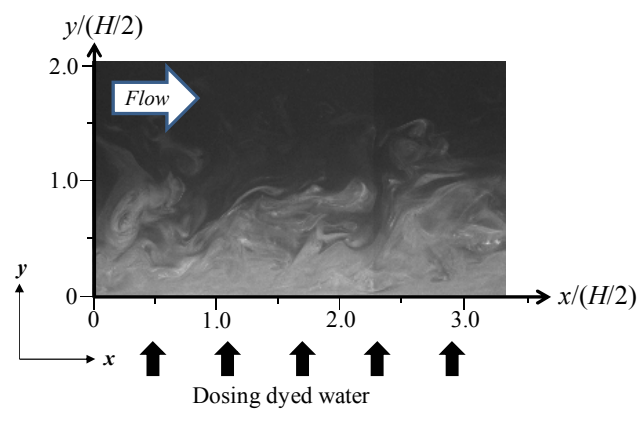

(a)

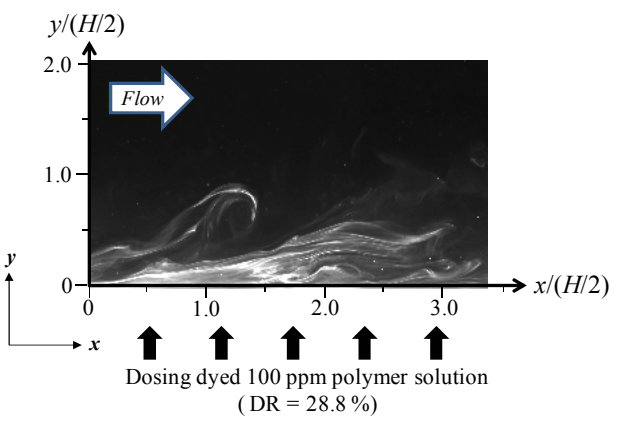

(b)

Fig. 14 Instantaneous PLIF images of the channel flow: (a) with dosing of dyed water, and (b) with dosing of $100 \mathrm{ppm}$ dyed polymer solution $(\mathrm{DR}=28.8 \%)$.

Figure 12 shows the relationship between Sherwood number and drag reduction rate for Reynolds numbers of 20000 and 40000 . For this figure, we calculated the local drag reduction rate and the Sherwood number under all dosing conditions at three positions from the leading edge of the dosing wall $(x=250,800$, and $1350 \mathrm{~mm})$. This figure clearly indicates that the Sherwood number decreases linearly with increasing drag reduction rate for both Reynolds numbers. Furthermore, this relationship is not affected by the dosing conditions. The results shown in Figs. 11 and 12 mean that, when the polymer solution is dosed from the wall, the polymer is accumulated and turbulent diffusion is suppressed downstream because the polymer solution is provided continuously from the wall to the downstream region. As a result of this suppression of the turbulent diffusion, drag reduction occurs and increases downstream, as shown in Fig. 6. Finally, we calculate the Stanton number $(S t)$. The Stanton number is defined by the following equation:

$$
S t=\frac{S h}{R e \cdot S c}
$$

Figure 13 shows the relationship between Stanton number and drag reduction rate. As shown in this figure, the drag reduction shows a linear relationship with the Stanton number, irrespective of the flow condition and dosing condition. Such a universal relationship, which does not depend on dosing condition (i.e. polymer concentration and dosing rate) and flow condition, suggests that the drag reduction phenomenon can be explained in various cases. This may be of great importance for investigating drag-reducing flow with dosed polymer solution from a wall.

\subsection{Instantaneous PLIF images}

Figure 14 shows the instantaneous PLIF images of the channel flow with (a) dosing of dyed water and (b) dosing of dyed $100 \mathrm{ppm}$ polymer solution $(D R=28.8 \%)$ at $R e=20000$. In the case of dosing of dyed water flow, it can be seen that dye is ejected from the wall to the center of the channel and well mixed. According to Adrian et al. (23), in turbulent flow, there are various scale vortexes and hairpin vortexes in the near-wall region, which cause sweep and ejection. An individual group of hairpin vortexes in the same streamwise velocity is called a "hairpin vortex packet" and a velocity difference exists between each packet. The boundary on this velocity difference is called the shear layer. Consequently, these structures cause an increase of skin frictional drag. The existence of this turbulent structure can be seen abstractedly in Fig. 14(a). In contrast, these vortexes are largely suppressed by dosing of $100 \mathrm{ppm}$ polymer solution. The dye is only distributed in the near-wall region. The pattern is streaky and extended in the streamwise direction. This indicates that the momentum transport to the wall-normal direction is largely suppressed by 


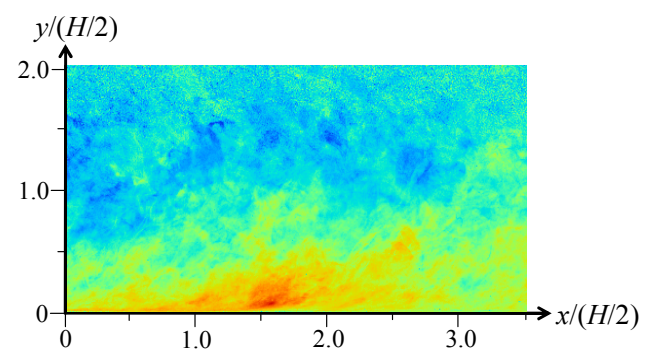

(a)

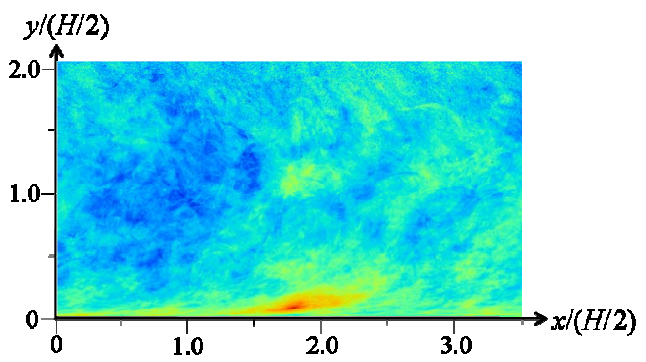

(b)

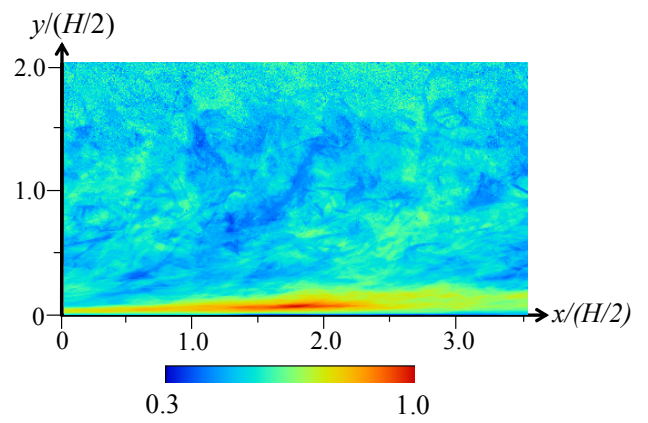

(c)

Fig. 15. Two-dimensional two-point correlations of the channel flow: (a) with dosing of dyed water and (b) with dosing of $50 \mathrm{ppm}$ dyed polymer solution $(D R=26.1 \%)$, and (c) with dosing of $200 \mathrm{ppm}$ dyed polymer solution $(D R=37.6 \%)$.

the dosed polymer solution. In our previous study ${ }^{(17)}$, we measured the velocity field in an $x-y$ plane by PIV with dosing $100 \mathrm{ppm}$ polymer solution at $2.0 \mathrm{~L} / \mathrm{min}$ (c.f. This study; 100 ppm polymer solution at $3.0 \mathrm{~L} / \mathrm{min}$ ). The results show that the drag reduction rate was almost same level, and the peak of the Reynolds shear stress significantly decreased. Particularly, Reynolds shear stress near the dosing wall for $y /(H / 2)<0.4$ decreased by about $35 \%$ in this case. Therefore, the suppression of the turbulent diffusion confirmed by the instantaneous PLIF image (Fig. 14(b)) has a close relationship with the suppression of the Reynolds shear stress.

On the other hand, in the case of the slot injection, Poreh and Hsu ${ }^{(20)}$ categorized the injected concentration distribution across the boundary layer that evolved in the streamwise direction into the following four regions: (i) Initial stage: the injected polymer exists within the sub-layer; (ii) Intermediate stage: the polymer lies within a layer thinner than the boundary layer thickness but wider than the buffer layer; (iii) Transition stage: the polymer is diffused throughout most of the turbulent boundary layer; and (iv) Final stage: polymer concentration becomes uniform in the flow. In addition, they reported that the region in which drag reduction develops (as mentioned in §3.1) corresponds to the initial stage and the intermediate stage. In the wall dosing method, almost the same tendency was obtained.

\subsection{Two-dimensional two-point correlation}

In order to investigate the diffusion in the near-wall region in more detail, we calculated two-dimensional two-point correlations of the concentration field for the water flow (dosed dyed water) and for the drag-reducing flow with dosed polymer solution. Two-dimensional two-point correlations are very useful for illustrating the structures statically in the flow. The two-dimensional two-point correlation of the concentration field calculated by the instantaneous PLIF is defined as follows: 


$$
R_{C}(\Delta x, \Delta y)=\frac{C^{\prime}(x, y) C^{\prime}(x+\Delta x, y+\Delta y)}{C_{r m s}(x, y) C_{r m s}(x+\Delta x, y+\Delta y)}
$$

Figure 15 shows the two-dimensional two-point correlation of the channel flow with (a) dosing of dyed water, and the drag-reducing channel flow with (b) dosing of $25 \mathrm{ppm}$ polymer solution $(D R=26.1 \%)$ and (c) dosing of $200 \mathrm{ppm}$ polymer solution $(D R=28.8 \%)$. The standard position was set to $x /(H / 2)=1.9$ and $y /(H / 2)=0.1$.

In the case of the water flow analyzed by dosing of dyed water, it can be seen that dyed water was well diffused to the center of the channel. In contrast, in the case of the drag-reducing flow with dosing of $25 \mathrm{ppm}$ polymer solution, since the turbulent diffusion was suppressed by polymer addition, it can be seen that the region where the high correlation extender toward the streamwise direction settled near the dosing wall and was inclined to the channel wall. Moreover, when more concentrated polymer solution (i.e. 200 ppm) was dosed into the channel flow, the high-correlation region settled more strongly near the dosing wall and the scale of the high-correlation region became large. These results mean that the turbulent diffusion was suppressed more strongly upon dosing with more condensed polymer solution. Consequently, drag reduction became large when the high-concentration polymer solution was dosed into the channel flow. The same tendency was also obtained by the two-dimensional two-point correlation of the velocity field in our previous PIV measurement ${ }^{(17)}$.

\section{Conclusion}

In this study, we investigated the relationship between mass transfer in the near-dosing-wall region and turbulent drag reduction by dosing polymer solution from whole surface of the channel wall. We measured the mass transfer qualitatively and quantitatively by two methods. Qualitative measurement was by PLIF. In contrast, quantitative measurement was that Sampling method. The main conclusions were drawn as follows:

1. The drag reduction rate develops downstream from the leading edge of the dosing wall. About $29 \%$ of the maximum drag reduction was obtained with wall dosing method at the position of $1350 \mathrm{~mm}$ downstream of the leading edge of the dosing wall.

2. The results of the streamwise development of mean concentration profile measured by the Sampling method show the high polymer concentration layer in the downstream direction extends into the outer region, the polymer concentration in the near-wall region still becomes higher downstream owing to the accumulation of polymer solution along the flow direction.

3. In the case of water flow, diffusion is strong near a wall and becomes weak away from the wall. In contrast, in the case of the drag-reducing flow, diffusion is largely suppressed in the near wall.

4. The suppression of the turbulent diffusion becomes strong with dosing higher concentration polymer solution. Consequently, drag reduction becomes large when the high concentration polymer solution was dosed into the channel flow under the range of the polymer concentration in our experimental conditions.

\section{Acknowledgments}

This study was carried out under the supports of the Fundamental Research Developing Associate for Shipbuilding and Offshore (REDAS) and the New Energy and Industrial Technology Development Organization (NEDO). We would like to thank Mr. E. Yoshikawa of the Chugoku Marine Paints, Ltd. for the supporting in this study. 


\section{References}

(1) Toms, B. A., Some observations on the flow of linear polymer solutions through straight tubes at large Reynolds numbers, Proceedings of 1st International Congress on Rheology, Amsterdam, (1948), pp. 135-141.

(2) Burger, E. D. and Munk, W. R., Flow increase in the Trans Alaska Pipeline through use of a polymeric drag-reducing additive, Journal of Petroleum Technology, Vol. 34 (1982), pp. 377-386.

(3) Gyr. A., and Bewersdorff, H. W., Drag reduction of turbulent flows by additives, Kluwer academic publishers, Dordrecht, (1995), Chap. 1 and 5.

(4) Virk, P. S., Merrill, E. W., Mickley, H. S., Smith, K. A. and Mollo-Christensen, E. L., The Toms phenomenon: turbulent pipe flow of dilute polymer solutions, Journal of Fluid Mechanics. Vol. 30 (1967), pp. 305-328.

(5) Virk, P. S., The ultimate asymptote and mean flow structure in Toms phenomenon, ASME Journal of Applied Mechanics, Vol. 37 (1970), pp. 480- 493.

(6) Kulik, V. M., Drag reduction change of polyethyleneoxide solutions in pipe flow, Experiments in Fluids, Vol. 31 (2001), pp. 558-566.

(7) Ptasinski, P. K., Nieuwstadt, F. T. M., Van den Brule. B. H. A. A. and Hulsen, M. A., Experiments in turbulent pipe flow with polymer additives at maximum drag reduction, Flow, Turbulence and Combustion, Vol. 66 (2001), pp.159-182.

(8) Min, T., Yoo, J. Y., Choi, H. and Joseph, D. D., Drag reduction by polymer additives in a turbulent channel flow, Journal of Fluid Mechanics, Vol. 486 (2003), pp.213-238.

(9) Kim, K. G., Li, C. F., Sureshkumar, R., Balachandar, S. and Adrian, R. J., Effects of polymer stress on eddy structures in drag-reduced turbulent channel flow, Journal of Fluid Mechanics. Vol. 584 (2007), pp.281-299.

(10) Tiederman, W. G., Luchik, T. S. and Bogard, D. G., Wall layer structure and drag reduction, Journal of Fluid Mechanics. Vol. 156 (1985), pp.419- 437.

(11) Walker, D. T. and Tiederman, W. G., The concentration field in a turbulent channel flow with polymer injection at the wall, Experiments in Fluids. Vol.8 (1989), pp.86-94.

(12) Vdovin, A. V. and Smol'yakov, A. V., Turbulent diffusion of polymers in a boundary layer, Journal of Applied Mechanics. Vol. 22 (1981), pp.526-531.

(13) Winkel, E. S., Oweis, G., Vanapalli, S. A., Dowling, D. R., Perlin, M., Solomon, J. and Ceccio, S. L., Friction drag reduction at high Reynolds numbers with wall injected polymer solutions, Proceedings of 26th Symposium. Naval Hydrodynamics, Rome, (2006), pp.17-22.

(14) Somandepalli, V. S. R., Hou, Y. S. and Mungal, M. G., Concentration flux measurement in a polymer drag-reduced turbulent boundary layer, Journal of Fluid Mechanics. Vol. 644 (2010), pp.281-319.

(15) Motozawa, M., Onose, Y., Sugita, S., Iwamoto, K., Ando, H., Senda, T. and Kawaguchi, Y., Experimental investigation on turbulent drag reduction with blowing polymer solution from the wall, Proceedings of 7th World Conference Experiment Heat Transfer, Fluid Mechanics and Thermodynamics, Krakow, (2009), pp.1025-1035

(16) Motozawa, M., Ito, T., Matsumoto, A., Ando, H., Ashida, T., Senda, T. and Kawaguchi, Y., Turbulent Drag Reduction by Polymer Containing Paint -Simultaneous Measurement of Skin Friction and Release Rate-, Proceedings of International Heat Transfer Conference (IHTC-14), Washington D. C., (2010), DVD-Rom.

(17) Motozawa, M., Ishitsuka, S., Iwamoto, K., Ando, H., Senda, T. and Kawaguchi, Y., Experimental Investigation on Turbulent Structure of Drag Reducing Channel Flow with Blowing Polymer Solution from the Wall, Flow, Turbulence and Combustion, Vol. 88 (2012), pp. 121-141.

(18) Shimadzu Homepage: http://www.shimadzu.com/ 
(19) Hou, Y. X., Somandepalli, V. S. R. and Mungal, M. G., Streamwise development of turbulent boundary-layer drag reduction with polymer injection, Journal of Fluid Mechanics. Vol. 597 (2008), pp.31-66.

(20) Poreh, M. and Hsu, K. S., Diffusion of drag reducing polymer in a turbulent boundary layer, Journal of Hydronautics, Vol. 6, No. 1 (1972), pp. 27-33.

(21) Kader, B. A., Temperature and Concentration Profiles in Fully Turbulent Boundary Layers, International Journal of Heat and Mass Transfer, Vol. 24, No. 9 (1981), pp. 1541-1544.

(22) Matthys, E. F., Heat transfer, drag reduction, and fluid characterization for turbulent flow of polymer solution: recent results and research needs, Journal of Non-Newtonian Fluid Mechanics, Vol. 38 (1991), pp. 313-342.

(23) Adrian, R. J., Meinhart, C. D. and Tomkins, C. D., Vortex organization in the outer region of the turbulent boundary layer, Journal of Fluid Mechanics, Vol. 422 (2000), pp.1-54. 